\title{
Resonantly Pumped Bright-Triplet Exciton Lasing in Cesium Lead Bromide Perovskites
}

Guanhua Ying, ${ }^{\dagger}$ Tristan Farrow, ${ }^{*}{ }^{\dagger}$ Atanu Jana, Hanbo Shao, Hyunsik Im, Vitaly Osokin, Seung Bin Baek, Mutibah Alanazi, Sanjit Karmakar, Manas Mukherjee,* Youngsin Park,* and Robert A. Taylor*

Cite This: ACS Photonics 2021, 8, 2699-2704

Read Online

ACCESS | Lلll Metrics \& More | 回 Article Recommendations | st Supporting Information

ABSTRACT: The surprising recent observation of highly emissive triplet-states in lead halide perovskites accounts for their orders-ofmagnitude brighter optical signals and high quantum efficiencies compared to other semiconductors. This makes them attractive for future optoelectronic applications, especially in bright low-threshold nanolasers. While nonresonantly pumped lasing from allinorganic lead-halide perovskites is now well-established as an attractive pathway to scalable low-power laser sources for nanooptoelectronics, here we showcase a resonant optical pumping scheme on a fast triplet-state in $\mathrm{Cs}_{\mathrm{PbBr}}$ nanocrystals. The scheme allows us to realize a polarized triplet-laser source that dramatically enhances the coherent signal by 1 order of magnitude while suppressing noncoherent contributions. The result is a source with

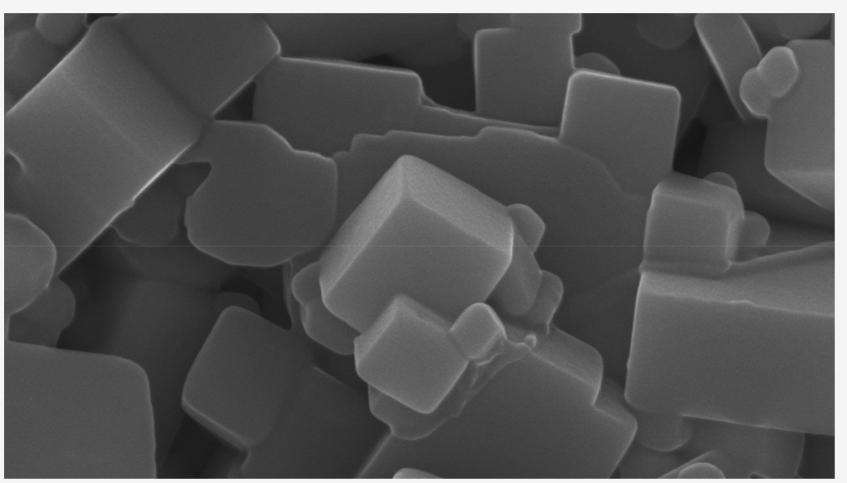
highly attractive technological characteristics, including a bright and polarized signal and a high stimulated-to-spontaneous emission signal contrast that can be filtered to enhance spectral purity. The emission is generated by pumping selectively on a weakly confined excitonic state with a Bohr radius $\sim 10 \mathrm{~nm}$ in the nanocrystals. The exciton fine-structure is revealed by the energy-splitting resulting from confinement in nanocrystals with tetragonal symmetry. We use a linear polarizer to resolve 2-fold nondegenerate sublevels in the triplet exciton and use photoluminescence excitation spectroscopy to determine the energy of the state before pumping it resonantly.

KEYWORDS: perovskites, nanocrystals, lasing, triplet exciton, photoluminescence

$\mathrm{W}$ hen it became apparent that triplet-excitons in allinorganic perovskites are optically active ${ }^{1,2}$ and, hence, do not suffer from intensity-quenching due to long-lived dark states seen in their hybrid counterparts, the idea that they could be exploited for bright polarized coherent emission above the lasing threshold followed naturally. Here we present a scheme to resonantly pump the triplets at fluences above lasing thresholds to realize coherent emission with bright polarized signals with low-incoherent background contributions. Optical transitions from triplet states are normally spinforbidden and result in long-lived dark states compared to emissive singlet states. This might appear to be problematic for the efficiency of optoelectronics given the three-to-one prevalence of triplets over singlets in a range of optical materials, including semiconductors and lead halide perovskites. ${ }^{1,4}$ Recent reports ${ }^{1,2}$ overturn this view by showing that the emission intensity from fast triplets in lead halide perovskite nanocrystals (PNCs) is not only bright, but accounts for why these materials can be up to $10 x^{3}$ brighter than other semiconductors. These are encouraging results for photovoltaics, single-photon sources, ${ }^{2}$ wavelength-tunable nanolasers, ${ }^{5-13}$ nonlinear ${ }^{14}$ and spintronic devices, ${ }^{15}$ and optoelectronic applications. ${ }^{11}$ Unlike in other semiconductors, the heavy ions in $\mathrm{CsPbBr}_{3}$ lead to strong coupling between the spin and orbital angular momenta of holes $\left(j_{\mathrm{h}}=1 / 2\right)$ and electrons (where $j_{\mathrm{e}}= \pm 1 / 2$, since the electronic state is doubly degenerate due to the stronger spin-orbit term in the conduction band ${ }^{4}$ ) such that only the total angular momentum $\left(J=j_{\mathrm{h}}+j_{\mathrm{e}}\right)$ is conserved. The spin-degeneracy is lifted when momenta of the electron and hole are mixed through an exchange interaction revealing the exciton's fine structure with distinct singlet $(J=0)$ and triplet $(J=1)$ states $^{1,2}$ (Figure 1a). While the exact mechanisms of symmetry-breaking and state energy reordering (where the triplet energy is pulled below the

Received: May 14, 2021

Published: August 27, 2021 
A

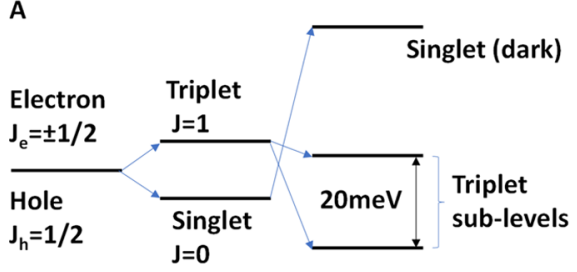

B

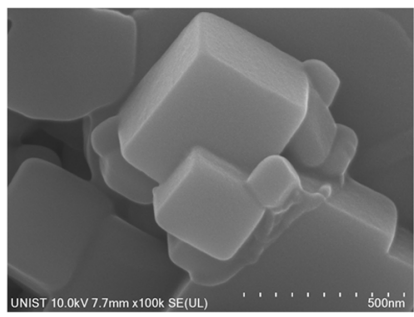

Figure 1. (A) Excitonic energy fine structure. (B) SEM of encapsulated $\mathrm{Cs}_{\mathrm{PbBr}}$ nanocrystals. (A) Energy fine structure in $\mathrm{CsPbBr}_{3}$. The presence of heavy ions in lead-halide perovskites leads to strong spin-orbit coupling while symmetry breaking produces a Rashba-type effect resulting in an inversion of the single and triplet energies and the lifting of the triplet degeneracy into dipole-allowed emissive triplet states. (B) Scanning electron microscope (SEM) image of monolithic Cs $4 \mathrm{PbBr}_{6}$ perovskite microcrystals. The microcrystals encapsulate regular arrays of $\mathrm{CsPbBr}_{3}$ nanocrystals with tetragonal symmetry (see Figure S1) and have dimensions ranging from 0.1 to $\sim 1 \mu \mathrm{m}$.
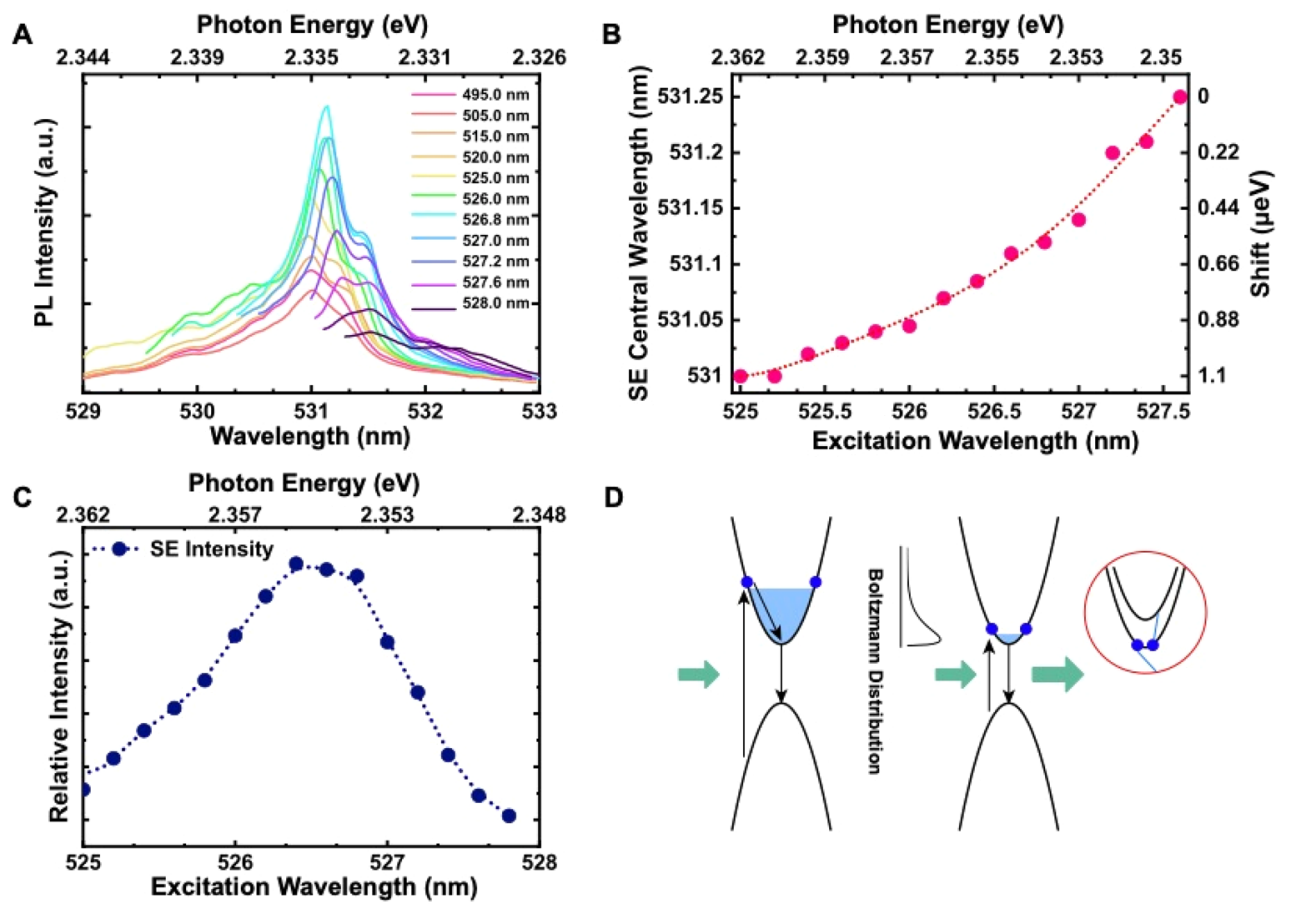

D

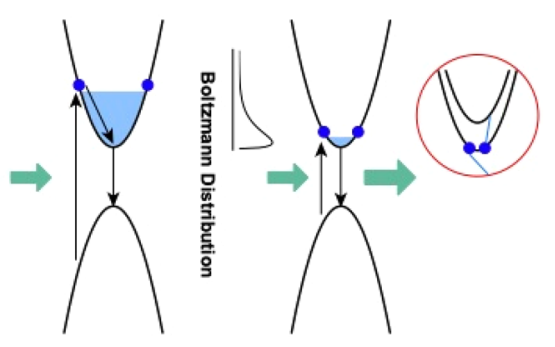

Figure 2. Photoluminescence spectra from $\mathrm{CsPbBr}_{3}$ PNCs as the pump wavelength is varied. (A) Excitation wavelength scan using a supercontinuum white laser in conjunction with an adjustable grating at $4 \mathrm{~K}$. The fluence can be controlled and the scan is only performed over a narrow wavelength range where the excitation intensity is kept constant. The figures on the right are the excitation wavelengths in $\mathrm{nm}$. (B) Red shift of the SE peak after the excitation wavelength exceeds the resonant pumping wavelength with the dotted line as an eye-guide. (C) Integrated SE intensity as a function of excitation wavelength. (D) In nonresonant excitation of an electron-hole pair, radiative and nonradiative phononscattering decay pathways can generate spontaneous emission resulting in an incoherent background signal on top of the stimulated signal. For excitations near the excitonic transition, the absorption coefficient increases, resulting in brighter photoluminescence and a suppression of the incoherent decay pathways as the intensity of the coherent signal increases. The inset shows an Auger mechanism when excess carriers concentrate in a small region.

singlet) producing a Rashba-type effect remain unclear, ${ }^{4}$ recent studies confirmed that triplets in lead halide PNCs become dipole-allowed and are bright. The triplet exciton resolves into three nondegenerate sublevels (with orthogonal linear dipoles) when quantum-confined in PNCs in the orthorhombic phase, and into two states in the tetragonal phase, but reverts to a degenerate triplet in the isomorphic (cubic) phase.

\section{RESULTS AND DISCUSSION}

In this work, we study colloidal PNCs (see Materials and Methods) in the tetragonal phase where the linear dipoles are polarized along two symmetry axes. We measure fine structure splitting by placing a linear polarizer into the optical path of the emission. The measurement is performed at cryogenic temperatures to resolve the splitting, since its energy is small compared to the thermal bath at room temperature.

The optical properties of our $\mathrm{Cs}_{\mathrm{PbBr}}$ nanocrystals are characterized via a fiber-based confocal microphotoluminescence $(\mu \mathrm{PL})$ setup with a tunable source for illumination. The source comprises a supercontinuum white pulsed laser operating at $78 \mathrm{MHz}$ with a pulse width of $10 \mathrm{ps}$ (SuperK Extreme, NKT Photonics) used together with a pair of controllable transmission gratings and an output slit to spectrally filter out a narrow band $(1.5-2 \mathrm{~nm})$ within the source wavelength range (Fianium fliter). The outgoing beam is then coupled into a multimode fiber for the purpose of beam-shaping before being focused through a $100 \times$ microscope objective onto a collection of microcrystals made of regular arrays of tetragonal perovskite nanocrystals with a 

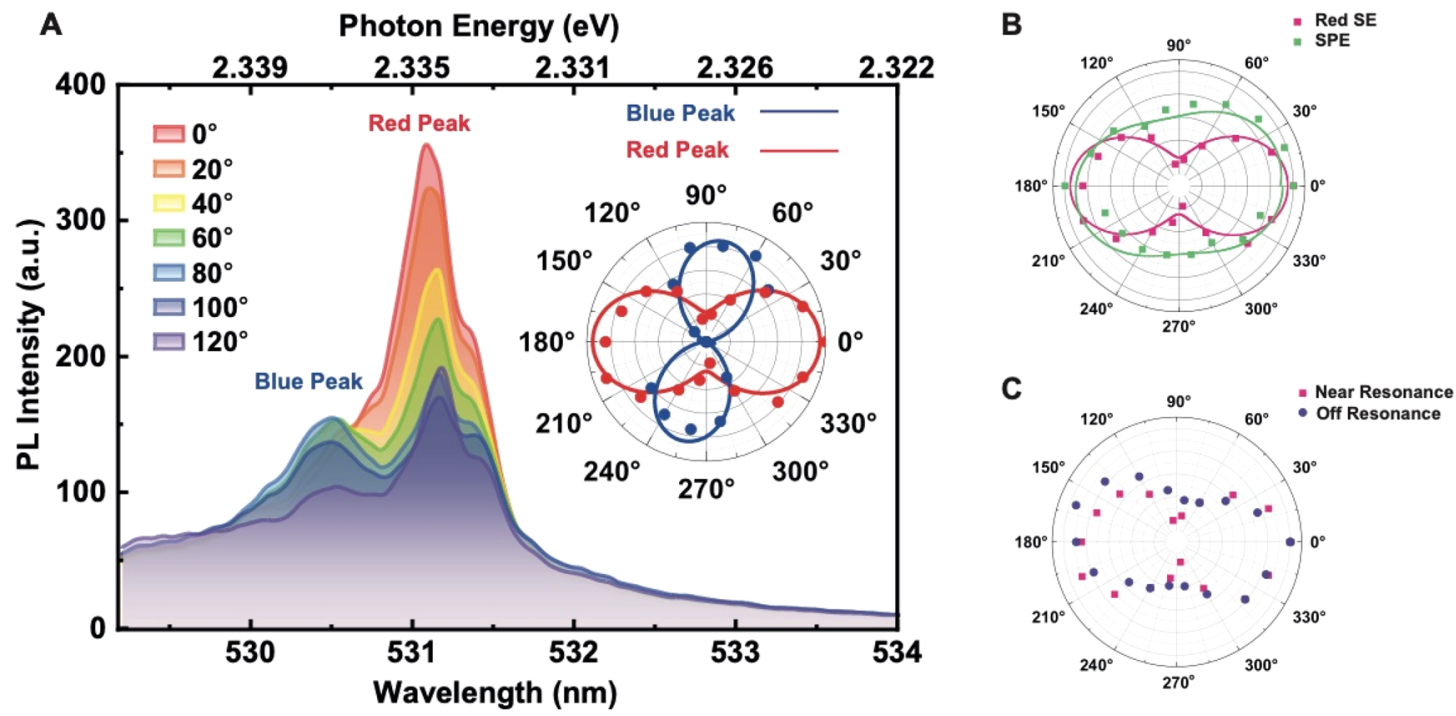

Figure 3. Polarization spectra of the $\mathrm{CsPbBr}_{3}$ nanocrystals. (A) Polarization-dependent PL spectrum of the PNCs measured at $4 \mathrm{~K}$ with an excitation power of $\sim 50 \mu \mathrm{W}$ and near-resonant laser excitation. The inset depicts a polar angle diagram of the two emission peaks using a linear polarizer in tandem with a half-wave plate to select a narrow polarization angle of the emission spectrum aligned with the spectrometer gratings. Each peak's maximum intensity on the polar plot is self-normalized. (B) A comparative study of the polarization-dependence revealed that the SE is markedly more polarized than the background signal. (C) Polarization-dependence of the on- and off-resonance polarization signals generated with a $2.505 \mathrm{eV}$ (495 nm wavelength) pump.

diffraction-limited spot size of $\sim 1.2 \mu \mathrm{m}$. The setup thus produces an excitation source with a spectrally narrow, spatially limited spot which allows the wavelength to be scanned continuously covering the full visible spectrum. The luminescence is then directed confocally to a spectrometer (spectral resolution of $\sim 700 \mu \mathrm{eV}$ ) via a multimode optical fiber of $25 \mu \mathrm{m}$ core size to limit the collection area to a spot with diameter $\sim 1 \mu \mathrm{m}$ at the sample. The signal is finally detected using a cooled charge coupled device (CCD) detector.

We investigated the on-resonance pumping regime of our $\mathrm{Cs} \mathrm{PbBr}_{3}$ nanocrystals by tuning the excitation energy gradually into quasi-resonance with the upper-level of a triplet state (Figure 1A) at $\sim 2.335 \mathrm{eV}(531 \mathrm{~nm})$, as determined by photoluminescence excitation spectroscopy (PLE), and a PLE spectrum is shown in Figure S3. Figure 2A shows a series of emission spectra as a function of excitation wavelength with the pump laser filtered spectrally at a pump power $\sim 50 \mu \mathrm{W}$, well above the lasing threshold at $4 \mathrm{~K}$. A red shift of the stimulated emission (SE) peak is observed Figure $2 \mathrm{~B}$ as the pump wavelength tunes into resonance with the excitonic transition. Since carriers excited by a near-resonant pump occupy lower-lying energy states closer to the band-edge, it follows that upon recombination their emission wavelength is red-shifted relative to that generated by states occupying higher energy levels. The relative intensity shows the maximum near the on-resonance excitation regime (Figure 2C). The Figure 2D highlights the multiple dynamical processes can take place during on-/off-resonant optical excitation.

To resolve the fine structure splitting of the state we placed a linear polarizer into the optical path of the emission. A polarization-dependent analysis was then performed on the photoluminescence (PL) with linear polarizer in tandem with a half-wave plate, which was used to select a narrow polarization angle of the emission spectrum by aligning it with the spectrometer grating. The polarization-resolved PL spectra in Figure $3 \mathrm{~A}$ show two peaks at 2.335 and $2.337 \mathrm{eV}$, revealing the fine-structure of the triplet sublevels separated by $2 \mathrm{meV}$. This is consistent with measurements ${ }^{1,2}$ for PNCs with edge lengths $\sim 10 \mathrm{~nm}$. Our time-resolved PL decay measurements (Figure S2) yield a lifetime of 49 ps for the stimulated emission and 268 ps for the spontaneous emission, consistent with a weakly confined exciton with a Bohr radius $\sim 10 \mathrm{~nm}^{1}$. We also observe a Stokes shift of $18 \mathrm{meV}$ (Figure S3) corresponding to the energy given up by the confined exciton to phonons in the crystal lattice combined with a broadening arising from the ensemble of nanocrystals involved in the emission and the fact that stimulated emission will always appear in regions of low optical loss. The magnitude of the effect corroborates values reported elsewhere ${ }^{16}$ for nanocrystals of comparable size.

The near-total extinction of the emission for certain polarizer angles shown in the polar plot inset in Figure 3A confirms that the emission is linearly polarized, and reveals the orthogonally polarized directions of two nondegenerate triplet sublevels under near-resonant excitation. The peaks are fitted with a Gaussian function (Figure S4). The integrated PL intensity is fitted using Malus's law, $I(\theta)=I_{\min }+I_{\max } \cos ^{2}(\theta-$ $\theta_{0}$ ), where $\theta$ is the of polarization angle and $I_{\min }$ and $I_{\max }$ are the minimum and maximum intensities, respectively. The degree of linear polarization, defined as $\left(I_{\max }-I_{\min }\right) /\left(I_{\max }+\right.$ $\left.I_{\min }\right)$, is calculated to be over $85 \%$ for the lower energy peak and $\sim 100 \%$ for the higher energy peak. As the polarizer angle is rotated, the emission intensity from each state increases as the other decreases until extinction when rotated through $90^{\circ}$. A comparative study of the polarization-dependence (Figure 3B) shows that the signal from the low-lying triplet state is significantly more polarized than the emission from higher energy states above the band gap. The on- /off-resonance emission associated with the red peak (Figure 3C) shows neartotal extinction when pumped resonantly, but slightly weaker intensity modulation by the polarizer when pumped above resonance with a $2.505 \mathrm{eV}$ (495 nm wavelength) pump. In general, resonant excitation reduces depolarization effects and in light of the observed Stokes shift with above-resonance pumping, a contributing factor to depolarization arises from 

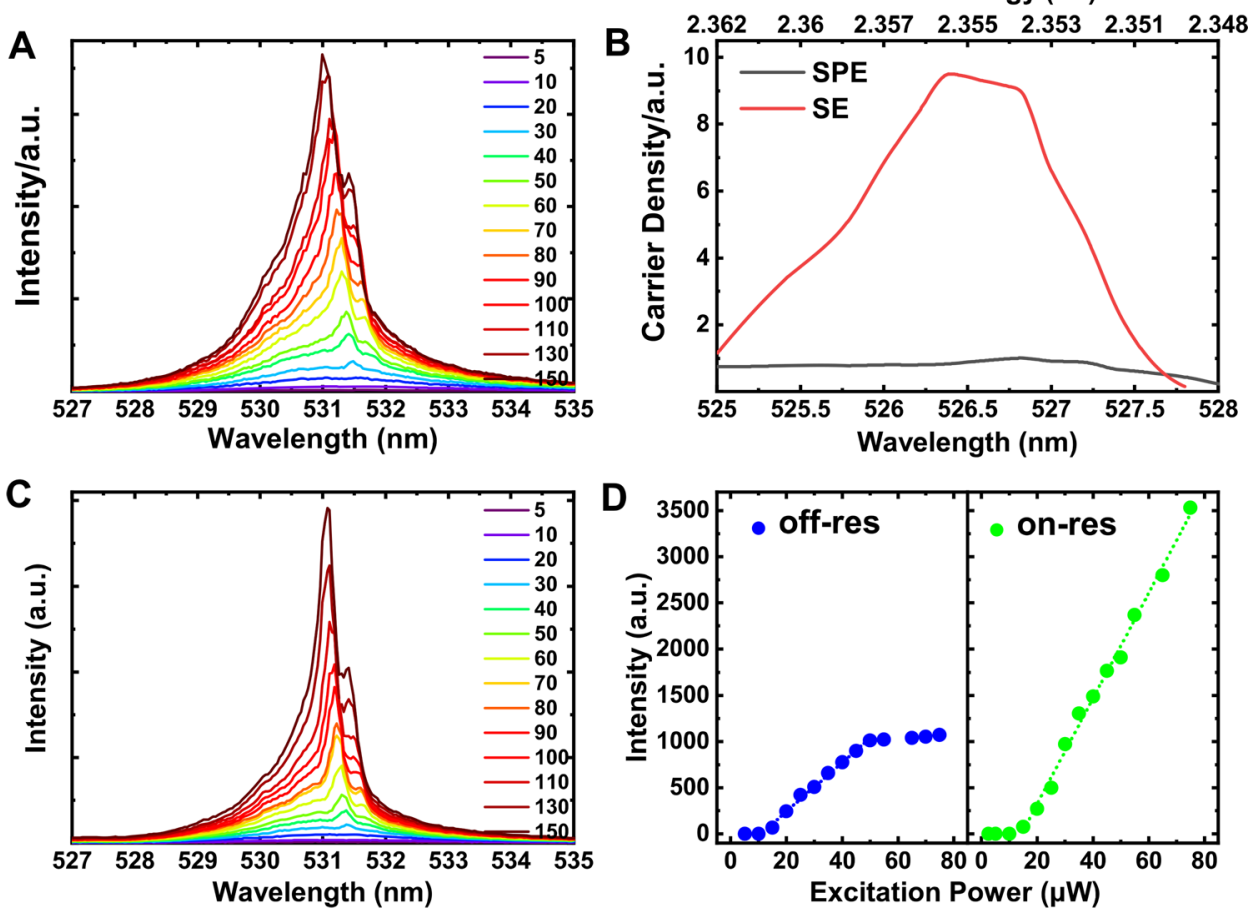

Figure 4. Excitation power-dependent PL spectra of the $\mathrm{CsPbBr}_{3}$ PNCs measured at $4 \mathrm{~K}$. (A) Intensity dependence on excitation power using an off-resonant optical pump $(495 \mathrm{~nm})$. The numbers relate to the excitation incident average power in $\mu \mathrm{W}$. (B) Nominal total carrier density compared to the carrier density contributing only to spontaneous emission (SPE) over the range of the wavelength scan. The difference between the two curves represents the integrated SE intensity. (C) Intensity dependence on excitation power using a near-resonant optical pump (526 nm). The excitation fluence is controlled to give the same density of pumping photons. Importantly, the contrast between the SE and SPE peaks is significantly higher even at near-threshold pumping. The numbers relate to the excitation incident average power in $\mu \mathrm{W}$. (D) Integrated SE intensity versus pump power for nonresonant (left panel) and near- resonant (right panel) pumping plotted on the same scale for emission intensity. Over the same range of pump-photon density, the near-resonant pumping regime produced a significantly steeper linear lasing intensity increase, while saturation is not reached even at the highest pumping fluence available. In the off-resonant pumping regime, a shallower linear intensity increase is observed, reaching saturation at $50 \mu \mathrm{W}$.

phonon scattering leading and a broadening of the confinement potential of the dipoles along the orthogonal polarization axes in the lattice. This is of technological relevance for applications where polarization-encoding of information depends on efficiently resolving the polarization basis, such as in single-photon source for quantum key distribution.

Figure $4 \mathrm{~A}$ shows the $\mu \mathrm{PL}$ spectra from $\mathrm{CsPbBr}_{3}$ PNCs illuminated at increasing excitation powers with an off-resonant pulse with an excitation energy of $2.505 \mathrm{eV}$ (495 nm). The PL peak around $531 \mathrm{~nm}$ falls within the well-known emission wavelength range of $\mathrm{CsPbBr}_{3}$ crystals at $4 \mathrm{~K}^{5-7}$ Since the wavelength of the pump-photons is tuned above resonance, $\mathrm{PL}$ is generated by carriers above the bandgap energy. Below excitation powers $\sim 10 \mu \mathrm{W}$, only a broad peak with fwhm (full at half-maximum $)<4 \mathrm{~nm}(18 \mathrm{meV})$ is visible, corresponding to spontaneous emission (SPE) due to incoherent recombination. The position and intensity of the peak was inferred from Gaussian fitting (Figure S4). As the excitation power increases, a sharp peak emerges and dominates the spectrum. This is attributed to stimulated emission (SE), corroborated by a characteristic S-shaped curve for the lasing intensitydependence on pump fluence (Figure 4D) above a threshold of $15 \mu \mathrm{W}\left(\sim 23 \mathrm{~mJ} / \mathrm{cm}^{2}\right)$ for lasing onset. Ultralow thresholds have been demonstrated with PNC lasers down to $220 \mathrm{~nJ} / \mathrm{cm}^{2}$ in pulsed ${ }^{17}$ and continuous-wave operation modes at room temperature, ${ }^{18}$ underscoring the technological viability of leadhalide perovskites as gain media for low-threshold lasers. ${ }^{19,20}$
We note a shift of the emission wavelength to higher energy with increasing pump power. This blue-shift is due to the recombination of states with energies above the extrema of the band-edges. At high pump fluences, these states are quickly repopulated, and as the pump power increases, higher-energy states lying further away from the band edges are populated. These hot carriers thermalize by scattering with phonons and other hot carriers until they reach the crystal temperature with energies characterized by a Boltzmann distribution, resulting in the observed blue-shift of the emission. ${ }^{21-23}$

As we approach the resonant pumping energy, the intensity of the lasing signal increases markedly relative to the incoherent signal. Conversely, we see a decrease of the lasing intensity as the pump-photon energy drops below the resonant energy, as expected. In Figure 4B we compare the relative carrier density contributing to the SE and SPE components of the emission as a function of pump wavelength. We note a marked increase in the proportion of carriers participating in $\mathrm{SE}$ as we near resonance, suggesting that incoherent decay pathways are suppressed. As the pump-energy nears resonance, incident photons scatter coherently with carriers occupying the lowest energy level close to the band-edge and generate a signal via SE. In the nonresonant excitation regime, carriers excited well above the band edge can also undergo SE, albeit many will decay via fast phonon-mediated relaxation, which detunes them from resonance with the pump. Thus, the fraction of carriers participating in $\mathrm{SE}$ is lower in the 
nonresonant regime. Because resonant pumping generates carriers near the band edge only, it suppresses the multiple incoherent decay pathways available to carriers in higherenergy levels. Hence it reduces incoherent contributions to the signal while enhancing lasing intensity and spectral purity. We show this explicitly in Figure 4D and measure a 10-fold enhancement of the lasing intensity on-resonance, while the SPE emission intensity increases only by $30 \%$. The fact that the on-resonance intensity continues to rise linearly with excitation power up to the maximum excitation power available at this energy $(80 \mu \mathrm{W})$ indicates that heating effects are not important over the range of powers used and little degradation of the signal is seen for long excitation times indicating that these systems would be suitable for real-word applications.

\section{CONCLUSIONS}

We demonstrated that resonant pumping on a bright triplet state in $\mathrm{CsPbBr}_{3}$ produces a laser source with highly desirable technological characteristics, including a low-threshold lasing onset, a bright polarized signal, and a high SE to SPE signal contrast that can be filtered to enhance spectral purity.

\section{MATERIALS AND METHOD}

Laser System and PL. The incident laser power on the $\mathrm{Cs} \mathrm{PbBr}_{3}$ surface ranged from $100 \mathrm{nW}$ to a few hundred $\mu \mathrm{W}$. The sample was mounted in a continuous-flow helium cryostat, allowing the temperature to be controlled accurately from $4 \mathrm{~K}$ to room temperature. Measurements are performed at cryogenic temperatures to resolve the triplet splitting, since its energy is small compared to the thermal bath at room temperature.

Optical Photoluminescence and Coherent Measurements. A colloidal solution of $\mathrm{CsPbBr}_{3}$ was dispersed on a quartz substrate. The optical properties of the $\mathrm{CsPbBr}_{3}$ nanocrystals were characterized using a fiber-based confocal microphotoluminescence ( $\mu \mathrm{PL})$ setup with a tunable pump source for photoluminescence excitation (PLE) spectroscopy. We used a supercontinuum white pulsed laser operating at 78 $\mathrm{MHz}$ with a pulse width of 10 ps (SuperK Extreme, NKT Photonics) for the source in conjunction with a controllable transmission grating and an output slit to spectrally filter a narrow band $(1.5-2 \mathrm{~nm})$ within the pump's wavelength range. A Picoharp time-correlated single photon counting system was used in conjunction with a photomultiplier for the timeresolved measurements.

Sample Preparation. Reagents. $\mathrm{Cs}_{2} \mathrm{CO}_{3}$ (99\%), $\mathrm{PbBr}_{2}$ (98\%), oleic acid (OA, $\geq 99 \%)$, 1-octadecene (ODE, tech., 90\%), $\mathrm{HBr}$ (ACS reagent, 48\%), octylamine (OAm, 99\%), and diethyl ether (99\%) were purchased from Sigma-Aldrich. Octylammonium bromide (OABr) was prepared according the previously reported method. ${ }^{22}$

Synthesis of Colloidal $\mathrm{Cs} \mathrm{PbBr}_{3}$ Tetragonal Nanocrystals Inside $\mathrm{Cs}_{4} \mathrm{PbBr}_{6}$ Microcrystals. In a typical synthesis, $\mathrm{Cs}_{2} \mathrm{CO}_{3}$ $(0.0325 \mathrm{~g}, 0.1 \mathrm{mmol})$ was dissolved in $2 \mathrm{~mL}$ of ODE and $1 \mathrm{~mL}$ of $\mathrm{OA}$ in a $15 \mathrm{~mL}$ glass vial under stirring conditions. The solution was dried for $1 \mathrm{~h}$ at $120{ }^{\circ} \mathrm{C}$ until all $\mathrm{Cs}_{2} \mathrm{CO}_{3}$ reacted with OA. $\mathrm{Cs}_{2} \mathrm{CO}_{3}$ reacts with $\mathrm{OA}$ to form Cs-oleate, $\mathrm{CO}_{2}$, and $\mathrm{H}_{2} \mathrm{O}$. At high temperature, both $\mathrm{CO}_{2}$ and $\mathrm{H}_{2} \mathrm{O}$ are evaporated. The solution was kept at $150{ }^{\circ} \mathrm{C}$ to avoid solidification. In a separate vial, $2 \mathrm{~mL}$ of ODE, $\mathrm{OAmBr}(0.042 \mathrm{~g}, 0.2 \mathrm{mmol})$, and $\mathrm{PbBr}_{2}(0.073 \mathrm{~g}, 0.2 \mathrm{mmol})$ and $1 \mathrm{~mL}$ of DMF were heated at $120{ }^{\circ} \mathrm{C}$ in open-air. Pure $\mathrm{OAm}$ is detrimental to the nanocrystal surface, as it may exist in dynamic equilibrium with the OA. ${ }^{23}$ Instead of pure OAm, here we have introduced bromide ammonium salt, $\mathrm{OABr}$ for synthesis of the PNCs. Here $3 \mathrm{~mL}$ of Cs-oleate solution was injected quickly into the lead precursor solution. After cooling to room temperature in ambient conditions, the crude solution was centrifuged immediately at $5000 \mathrm{rpm}$ for $5 \mathrm{~min}$. Then, the supernatant was removed, and the precipitate was dried at $60{ }^{\circ} \mathrm{C}$ for further use. Generally, the $\mathrm{Cs} \mathrm{PbBr}_{3}$ PNCs were synthesized using oleic acid and oleylamine, which have the same number of carbon atoms, and both the surface-passivating ligands maintain the homogeneous distribution of cubic-sized PNCs. ${ }^{24}$ In our case, we have used $\mathrm{OABr}$, which has eight carbon atoms, and the combination of short-chain $\mathrm{OABr}$ and long-chain $\mathrm{OA}$ results in a large quantity of $\mathrm{CsPbBr}_{3}$ PNCs embedded regularly in $\mathrm{Cs}_{4} \mathrm{PbBr}_{6}$ microcrystals with dimensions ranging from 0.1 to $\sim 1 \mu \mathrm{m}$.

\section{ASSOCIATED CONTENT}

\section{Supporting Information}

The Supporting Information is available free of charge at https://pubs.acs.org/doi/10.1021/acsphotonics.1c00720.

TEM images of the nanocrystals, a time-resolved emission spectrum, PLE and PL plots, and fitting data (PDF)

\section{AUTHOR INFORMATION}

\section{Corresponding Authors}

Tristan Farrow - Clarendon Laboratory, Department of Physics, University of Oxford, Oxford OX1 3PU, United Kingdom; Centre for Quantum Technologies, National University of Singapore, Singapore 117543, Singapore; (1) orcid.org/0000-0002-2393-9745;

Email: tristan.farrow@cantab.net

Manas Mukherjee - Centre for Quantum Technologies, National University of Singapore, Singapore 117543, Singapore; Email: cqtmukhe@nus.edu.sg

Youngsin Park - School of Natural Science, Ulsan National Institute of Science and Technology, Ulsan 44919, Korea; 다이.org/0000-0002-1789-750X; Email: ysinpark@ unist.ac.kr

Robert A. Taylor - Clarendon Laboratory, Department of Physics, University of Oxford, Oxford OX1 3PU, United Kingdom; 이잉.org/0000-0003-2578-9645; Email: robert.taylor@physics.ox.ac.uk

\section{Authors}

Guanhua Ying - Clarendon Laboratory, Department of Physics, University of Oxford, Oxford OX1 3PU, United Kingdom

Atanu Jana - Division of Physics and Semiconductor, Dongguk University, Seoul 04620, Korea; 다이.org/0000-00016566-0438

Hanbo Shao - State Key Laboratory of Mechanics and Control of Mechanical Structures, Nanjing University of Aeronautics and Astronautics, Nanjing, Jiangsu 210016, China

Hyunsik Im - Division of Physics and Semiconductor, Dongguk University, Seoul 04620, Korea

Vitaly Osokin - Clarendon Laboratory, Department of Physics, University of Oxford, Oxford OX1 3PU, United Kingdom 
Seung Bin Baek - School of Natural Science, Ulsan National Institute of Science and Technology, Ulsan 44919, Korea

Mutibah Alanazi - Clarendon Laboratory, Department of Physics, University of Oxford, Oxford OX1 3PU, United Kingdom

Sanjit Karmakar - Centre for Quantum Technologies, National University of Singapore, Singapore 117543, Singapore

Complete contact information is available at: https://pubs.acs.org/10.1021/acsphotonics.1c00720

\section{Author Contributions}

${ }^{\dagger}$ Co-first authors.

\section{Notes}

The authors declare no competing financial interest.

\section{ACKNOWLEDGMENTS}

T.F. acknowledges support from the Centre for Quantum Technologies (NUS) Exploratory Fund. T.F. and M.M. acknowledge support from the Singapore Ministry of Education and the National Research Foundation, Y.P. acknowledges support from the National Research Foundation of Korea (NRF) grant funded by the Korean government (MSIT; 2018R1D1A1B07043676 and 2021R1A2C1006113). The Oxford authors acknowledge support from EPSRC and the Oxford-ShanghaiTech collaboration project. The data related to the figures are freely available at: 10.5287/ bodleian:E9gbwmVNk

\section{REFERENCES}

(1) Becker, M. A.; Vaxenburg, R.; Nedelcu, G.; Sercel, P. C.; Shabaev, A.; Mehl, M. J.; Michopoulos, J. G.; Lambrakos, S. G.; Bernstein, N.; Lyons, J. L.; Stöferle, T.; Mahrt, R. F.; Kovalenko, M. V.; Norris, D. J.; Rainò, G.; Efros, A. L. Bright triplet excitons in caesium lead halide perovskites. Nature 2018, 553, 189-193.

(2) Utzat, H.; Sun, W.; Kaplan, A. E. K.; Krieg, F.; Ginterseder, M.; Spokoyny, B.; Klein, N. D.; Shulenberger, K. E.; Perkinson, C. F.; Kovalenko, M. V.; Bawendi, M. G. Coherent single-photon emission from colloidal lead halide perovskite quantum dots. Science 2019, 363, 1068-1072.

(3) Saba, M. Rule-breaking perovskites. Nature 2018, 553, 163-164.

(4) Stranks, S. D.; Plochocka, P. The influence of the Rashba effect. Nat. Mater. 2018, 17, 381-382.

(5) Swarnkar, A.; Chulliyil, R.; Ravi, V. K.; Irfanullah, M.; Chowdhury, A.; Nag, A. Colloidal Cs $\mathrm{PbBr}_{3}$ perovskite nanocrystals: Luminescence beyond traditional quantum dots. Angew. Chem. 2015, 127, 15644-15648.

(6) Rainò, G.; Nedelcu, G.; Protesescu, L.; Bodnarchuk, M. I.; Kovalenko, M. V.; Mahrt, R. F.; Stöferle, T. Single cesium lead halide perovskite nanocrystals at low temperature: fast single-photon emission, reduced blinking, and exciton fine structure. ACS Nano 2016, 10, 2485-2490.

(7) Makarov, N. S.; Guo, S.; Isaienko, O.; Liu, W.; Robel, I.; Klimov, V. I. Spectral and dynamical properties of single excitons, biexcitons, and trions in cesium-lead-halide perovskite quantum dots. Nano Lett. 2016, 16, 2349-2362.

(8) Park, Y.; Ying, G.; Jana, A.; Osokin, V.; Kocher, C. C.; Farrow, T.; Taylor, R. A.; Kim, K. S. Coarse and fine-tuning of lasing transverse electromagnetic modes in coupled all-inorganic perovskite quantum dots. Nano Res. 2021, 14, 108-113.

(9) Sutherland, B.; Sargent, E. Perovskite photonic sources. Nat. Photonics 2016, 10, 295-302.

(10) Veldhuis, S. A.; Boix, P. P.; Yantara, N.; Li, M.; Sum, T. C.; Mathews, N.; Mhaisalkar, S. G. Perovskite materials for light-emitting diodes and lasers. Adv. Mater. 2016, 28, 6804-6834.
(11) Kovalenko, M. V.; Protesescu, L.; Bodnarchuk, M. I. Properties and potential optoelectronic applications of lead halide perovskite nanocrystals. Science 2017, 358, 745-750.

(12) Chen, J.; Du, W.; Shi, J.; Li, M.; Wang, Y.; Zhang, Q.; Liu, X. Perovskite quantum dot lasers. Infomat 2020, 2, 170-183.

(13) De Giorgi, M. L.; Anni, M. Amplified spontaneous emission and lasing in lead halide perovskites: state of the art and perspectives. Appl. Sci. 2019, 9, 4591.

(14) Xu, J.; Li, X.; Xiong, J.; Yuan, C.; Semin, S.; Rasing, T.; Bu, X. H. Halide perovskites for nonlinear optics. Adv. Mater. 2020, 32, 1806736.

(15) Dong, Y.; Zhang, Y.; Li, X.; Feng, Y.; Zhang, H.; Xu, J. Chiral perovskites: promising materials toward next-generation optoelectronics. Small 2019, 15, 1970209.

(16) Brennan, M. C.; Forde, A.; Zhukovskyi, M.; Baublis, A. J.; Morozov, Y. V.; Zhang, S.; Zhang, Z.; Kilin, D. S.; Kuno, M. Universal size-dependent stokes shifts in lead halide perovskite nanocrystals. J. Phys. Chem. Lett. 2020, 11, 4937-4944.

(17) Zhu, H.; Fu, Y.; Meng, F.; Wu, X.; Gong, Z.; Ding, Q.; Gustafsson, M. V.; Trinh, M. T.; Jin, S.; Zhu, X. Y. Lead halide perovskite nanowire lasers with low lasing thresholds and high quality factors. Nat. Mater. 2015, 14, 636-642.

(18) Qin, C.; Sandanayaka, A. S. D.; Zhao, C.; Matsushima, T.; Zhang, D.; Fujihara, T.; Adachi, C. Stable room-temperature continuous-wave lasing in quasi-2D perovskite films. Nature 2020, $585,53-57$.

(19) Yakunin, S.; Protesescu, L.; Krieg, F.; Bodnarchuk, M. I.; Nedelcu, G.; Humer, M.; De Luca, G.; Fiebig, M.; Heiss, W.; Kovalenko, M. V. Low-threshold amplified spontaneous emission and lasing from colloidal nanocrystals of caesium lead halide perovskites. Nat. Commun. 2015, 6, 8056.

(20) Wu, K.; Park, Y. S.; Lim, J.; Klimov, V. I. Towards zerothreshold optical gain using charged semiconductor quantum dots. Nat. Nanotechnol. 2017, 12, 1140-1147.

(21) Zhou, C.; Zhong, Y.; Dong, H.; Zheng, W.; Tan, J.; Jie, Q.; Pan, A.; Zhang, L.; Xie, W. Cooperative excitonic quantum ensemble in perovskite-assembly superlattice microcavities. Nat. Commun. 2020, $11,329$.

(22) Fu, J.; Xu, Q.; Han, G.; Wu, B.; Huan, C. H. A.; Leek, M. L.; Sum, T. C. Hot carrier cooling mechanisms in halide perovskites. Nat. Commun. 2017, 8, 1300.

(23) Liu, Q.; Wang, Y.; Sui, N.; Wang, Y.; Chi, X.; Wang, Q.; Chen, Y.; Ji, W.; Zou, L.; Zhang, H. Exciton relaxation dynamics in photoexcited $\mathrm{CsPbI}_{3}$ perovskite nanocrystals. Sci. Rep. 2016, 6, 29442.

(24) Jana, A.; Mittal, M.; Singla, A.; Sapra, S. Solvent-free, mechanochemical syntheses of bulk trihalide perovskites and their nanoparticles. Chem. Commun. 2017, 53, 3046-3049. 